\title{
Tissue Engineering Applications in Medicine
}

\author{
Lara Tutunji* \\ Department of Pharmaceutics and Pharmaceutical Sciences, Jordan
}

*Corresponding author: Lara Tutunji, Department of Pharmaceutics and Pharmaceutical Sciences, Jordan

Submission: 眥 August 08, 2017; Published: 悳 September 06, 2017

\section{Editorial}

Tissue engineering (TE) is a rapidly growing, interdisciplinary field within health sciences that is devoted to manufacturing intact tissue and organ constructs by imitation natural biological development. TE is considered to be a subfield of regenerative medicine. Tissue engineering he field aims to regenerate whole biological components outside the body for eventual replacement therapy (i.e. implementation) via the manipulation of cells, natural of synthetic cell-supporting scaffold materials and bioactive molecules. The main use of tissue engineering is in all surgical subspecialties requiring viable transplantable body parts for the reconstruction of anatomic structures. Thus major issues in transplantation have been addresses such as a potentially inexhaustible source of tissue and organs as well circumventing the need for immune suppression by growing tissue from cells [1].

Tissue engineering can be used to develop functional constructs that can be used to reestablish, maintain, or improve the condition of injured body parts or tissues. This approach uses the patient's own cells to grow the tissue or organ required outside of the body using the patient's own cells; the engineered tissue or organ is then transplanted into the body, thus mimicking the native ECM binding sites and topography of the original tissue or organ.

Recent approaches toward tissue engineering have mainly focused on:

i. implanting newly created cells for the restoration of cellular structural network;

ii. exploring biomaterial scaffolds for endogenous cell infiltration, proliferation, and subsequent regeneration;

iii. A combination of (i) and (ii); and (iv) in situ tissue engineering by the incorporation of regenerative stimuli in the biomaterial scaffold [2].

Currently, tissue engineering is based on organ bioengineering where stem cells and the biomaterials used to generate cells, allow them to grow and proliferate are equally important [1]. "Functional tissue engineering" is a subset of the field of tissue engineering that was proposed by the US National Committee on Biomechanics over a decade ago in order to place more emphasis on the role of biomechanics and mechanobiologyin tissue repair and regeneration.
Novel biomaterials and material structures can provide functional biomechanical properties at the macro, micro, and nano-levels. The biomechanical and biological interactions between the stem cells and the scaffold may have significant influence on the long term success of engineered tissues. Once implanted, tissue-engineered constructs will be subjected to significant loads and deformations in vivo. Both the mechanical and biological consequences of in vivo loading must be understood to improve the success of engineered repairs and to develop appropriate rehabilitation protocols [3].

Since 2003, most tissue-engineered products have been marketed in the USA and European Union (EU) for the management of burns and wounds, and also for cartilage replacement. Research has also been performed into tissue engineering bone for treating craniofacial defects, cartilage for patients with osteoarthritis, and alveolar bone for patients with a cleft lip and palate, as well as for the regeneration of hair follicles, treatment for narrowing of the voice-box and upper windpipe, replacement corneas, and blood vessels for the treatment of cardiovascular conditions. Research has also focused on the development of biological bandages with antimicrobial peptide dendrimers that can be used to improve the treatment of burns without inducing any cytotoxicity and without altering the gene profile of progenitor cells. To establish the functionalized engineered tissue to recover the structural integrity of the damaged tissue or organ, three major components are necessary; the scaffold; the cells to be seeded; and the growth factors required in the growth medium [3].

Successful incorporation of dendritic scaffolds in tissue engineering has resulted from significant advances in the manufacturing of dendritic nano particles and improvements in the scaffold architecture. Several biomedical components utilizing dendrimers are now in preclinical and clinical trials, and it is clear from the research discussed here that the future for dendrimers and their associated scaffolds in tissue engineering and beyond is certainly bright [3].

The ethical issues that arise in tissue engineering research are complex and worthy of careful examination. Much issue engineering research is still in preclinical stages. Although ethical issues are often not addressed as they should be in research until human trials have begun, there are many issues worthy of consideration 
at the bench. These include: data integrity, responsible reporting, and dissemination of results, as well as ensuring that every study is designed and conducted so that it can yield results suitable to decide on the next research steps. It is important to realize that, at any stage, the results of well-designed and properly conducted research might not lead forward, but back or in a different direction than expected. It is also important to refine and expand knowledge at an earlier stage of the research or to explore and develop newly identified possibilities [4].

The use of animal models at preclinical stages remains vital to the success of tissue engineering research. Efforts are being taken to reduce the use of animals, but potential alternatives like computer modeling and body-on-a-chip organdie have significant limitations and require considerable further development. Researchers must consider the three Rs of animal research in this case-Reduce, Refine, and Replace. The choice of animal models and their humane and appropriate use helps to ensure that the research transition from animals to humans adheres to principle of "modest translational distance" described by Jonathan Kimmerlman. Translational distance (TD) refers to the number and size of inferential loops from animals to humans-in other words, it measures uncertainty [4]. In order to move from preclinical to first-in-human and other early stage trials, several questions must be answered in the affirmative [4]:

A. Has enough preclinical data collected from animal models before the researchers conduct further studies in humans?

B. Has enough been done to reduce the harm and increase the benefit to humans? This is of special importance in early stages of the research especially in humans.

C. Has the point of irreducible uncertainty been achieved? This question is important to know as there are always risks that are bound to occur and it is never possible to illuminate all the uncertainty concerning the occurrence of such risks.

D. Is the amount of irreducible uncertainty small enough so as to be able to ask human subjects and volunteers to take part the clinical research being conducted? This is a very important question to answer from the ethical point of view.

Two key considerations that relate the design of clinical trials to research ethics considerations are scientific validity and social value. A valid study is methodologically rigorous, designed, and powered to provide useful answers to questions it asks-including negative answers. A valuable study asks a socially meaningful and/or scientifically useful question. Value in research is usually defined as progressive value $\mathrm{p}$ that is, whether a study's results are able to move the line of research directly forward to the next phase. Researchers who recognize the broad applicability of study data and design translational research to take advantage of many different types of value can readily plan both to address what would otherwise simply be regarded as a failure of research progress and to enhance the value of successful research [4].

\section{References}

1. Katari RS, Peloso A, Orlando G (2014) Tissue Engineering. Advances in Surgery 48(1): 137-154.

2. Gorian B, Tekade M, Kesharwani P, Iyer AK, Kalias K, et al. (2017) The use of nanoscaffolds and dendrimers in tissue engineering. Drug Discovery Today 22(4): 652-664.

3. Guilak F, Baaijens FP (2014) Functional Tissue Engineering: Ten more years of progress. Journal of Biomechanics 47(9): 1931-1932.

4. Baker HB, McQuilling JP, King NMP (2016) Ethical Considerations in Tissue Engineering Research: Case Studies in Translation. Methods 99: 135-144. 